were assessed for demographic characteristics, disease duration, HLA-B27, DMARD and biological usage, biological switch ratio. Baseline disease activity was assessed with BASDAI, BASFI VAS (pain, fatigue and patients global assessment), ESR and CRP. Patients were compared according to having uveitis or not. Retention rate of adalimumab assessed by Kaplan-Meier survival analysis. Results: Total 350 (59.4\% male) AS patients analyzed. Mean age was 43 (12), mean disease duration and symptom duration were 10.5 (7.8) and 14.8 (9.6) years, respectively. 52 patients $(14.8 \%)$ had uveitis. Median adalimumab survival time according to having uveitis were $71.9(95 \% \mathrm{Cl} 25.4-118.6)$ months vs 36.4 $(95 \% \mathrm{Cl} 23.3-49.4)$ months (log-rank $\mathrm{p}=0.014)$ (figure). Patients with uveitis were more frequently male $(18.2 \%$ vs $9.8 \%, p=0.03)$, HLA-B27 positive $(75.0 \%$ vs $49.5 \%, p=0.022)$. Patients with uveitis had more frequently SpA family history, as well $(23.1 \%$ vs $10.8 \%, p=0.003)$. Age (49 (11) vs 41 (11) years, $p<0.001)$, disease duration $(14.4(10.1)$ vs $9.8(7.1)$ years, $p<0.001)$, and symptom duration (22 (12) vs 14 (8) years, $p<0.001$ ) were higher in patients with uveitis. Baseline and last visit disease activities were similar regarding to uveitis.

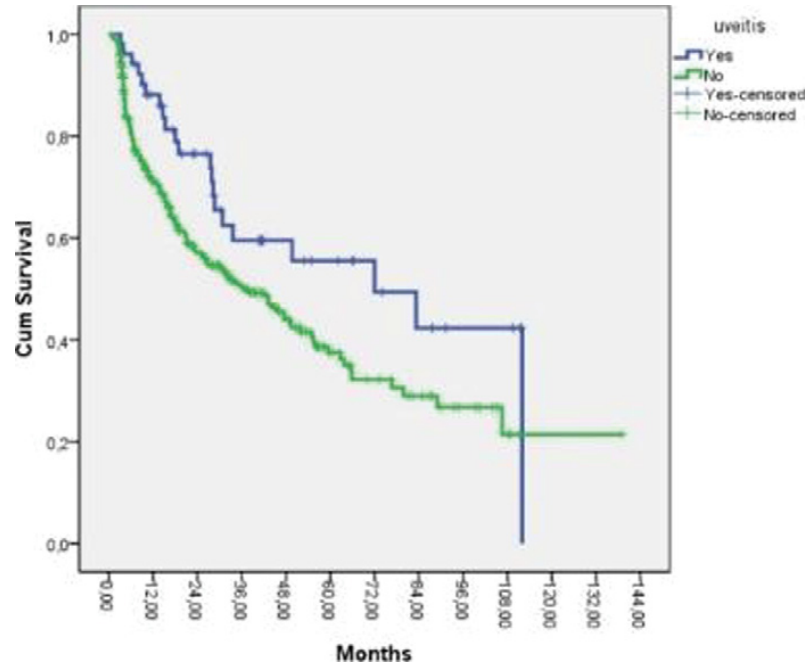

Conclusions: Determination of possible risk factors for retention of TNFi drugs is an important clinical problem for routine practise. It is well known that adalimumab is one of the treatment option for uveitis whether uveitis related with SpA or not. Our biological cohort supported that AS patients with uveitis had better adalimumab survival. For routine practise, adalimumab could be considered for AS patients with uveitis.

Disclosure of Interest: None declared

DOI: 10.1136/annrheumdis-2017-eular.4367

\section{AB0707 COMPARATIVE EFFECTIVENESS OF SECUKINUMAB AND GOLIMUMAB IN ANKYLOSING SPONDYLITIS ASSESSED BY MATCHING-ADJUSTED INDIRECT COMPARISON USING PIVOTAL PHASE 3 CLINICAL TRIAL DATA}

W. Maksymowych ${ }^{1}$, E. Choy ${ }^{2}$, Y. Yazici ${ }^{3}$, J. Walsh ${ }^{4}$, H. Thom ${ }^{5}$, C. Kalyvas ${ }^{6}$, T. Fox ${ }^{7}$, K. Gandhi ${ }^{8}$, S. Jugl ${ }^{7} .{ }^{1}$ University of Alberta, Edmonton, Canada; ${ }^{2}$ University of Cardiff, Cardiff, United Kingdom; ${ }^{3}$ New York University School of Medicine, New York; ${ }^{4}$ University of Utah, Salt Lake City, United States;

${ }^{5}$ University of Bristol, Bristol, United Kingdom; ${ }^{6} \mathrm{MAPI}$ Group, Houten,

Netherlands; ${ }^{7}$ Novartis Pharma AG, Basel, Switzerland; ${ }^{8}$ Novartis

Pharmaceuticals Corporation, East Hanover, United States

Background: No data are available from head-to-head RCTs between secukinumab $150 \mathrm{mg}$ (SEC; an anti-IL-17A) and golimumab $50 \mathrm{mg}$ (GOL; a TNFi) in patients with active ankylosing spondylitis (AS). Matching-Adjusted Indirect Comparison (MAIC) can be used to estimate comparative effectiveness and enables treatment outcomes to be compared across effectively balanced trial populations. MAIC is an established method in health technology assessments and NICE have published guidance on appropriate methodology, and especially for addressing imbalances in observed covariates between trials. ${ }^{1}$

Objectives: To assess the comparative effectiveness of SEC and GOL up to week 24 using MAIC with pooled individual patient data (IPD) from the RCTs MEASURE 1 (M1) and MEASURE 2 (M2) and published aggregate data from the RCT GO-RAISE.

Methods: Pooled M1 and M2 data were used to maximize the effective sample size (ESS) for SEC. IPD from the SEC arms of M1 and M2 ( $n=197)$ were weighted to match the published baseline characteristics of the GOL arm of GO-RAISE $(n=138)$. Placebo arms were matched in the same way; placeboadjusted comparisons were possible only until week 16 because patients could receive active treatment from this time onwards. Logistic regression was used to determine weights for age, sex, BASFI, disease duration, CRP and previous TNFi therapy. Recalculated outcomes from M1 and M2 (SEC, ESS=102; placebo, $E S S=81$ ) were compared with data from GO-RAISE (GOL, $n=138$; placebo, $\mathrm{n}=78$ ). Pairwise comparisons - reported as odds ratios (ORs [95\% Cls]) - were performed for ASAS 20, ASAS 40 and ASAS PR responses at nearest-equivalent time points across trials: week 12 (SEC)/14 (GOL), week 14 (GOL)/16 (SEC) and week 24 (SEC and GOL). Non-responder imputation (NRI) was available for all binary outcome data. Strict thresholds were avoided when interpreting $p$ values, in line with American Statistical Association 2016 guidance.

Results: There was no evidence of differences in ASAS 20 and ASAS 40 responses between SEC and GOL at weeks 12/14 and 14/16 (both placeboadjusted). At week 24, non-placebo-adjusted ASAS 20 and ASAS 40 responses using NRI were higher with SEC than GOL (OR [95\% Cl]: 1.58 [0.93-2.69], $p=0.089$ and 1.58 [0.94-2.64], $p=0.084$, respectively). There was no evidence of differences in ASAS PR responses between SEC and GOL at weeks 12/14, $14 / 16$ and 24 . A sensitivity analysis conducted after adding BASDAI score to the matching parameters yielded similar results.

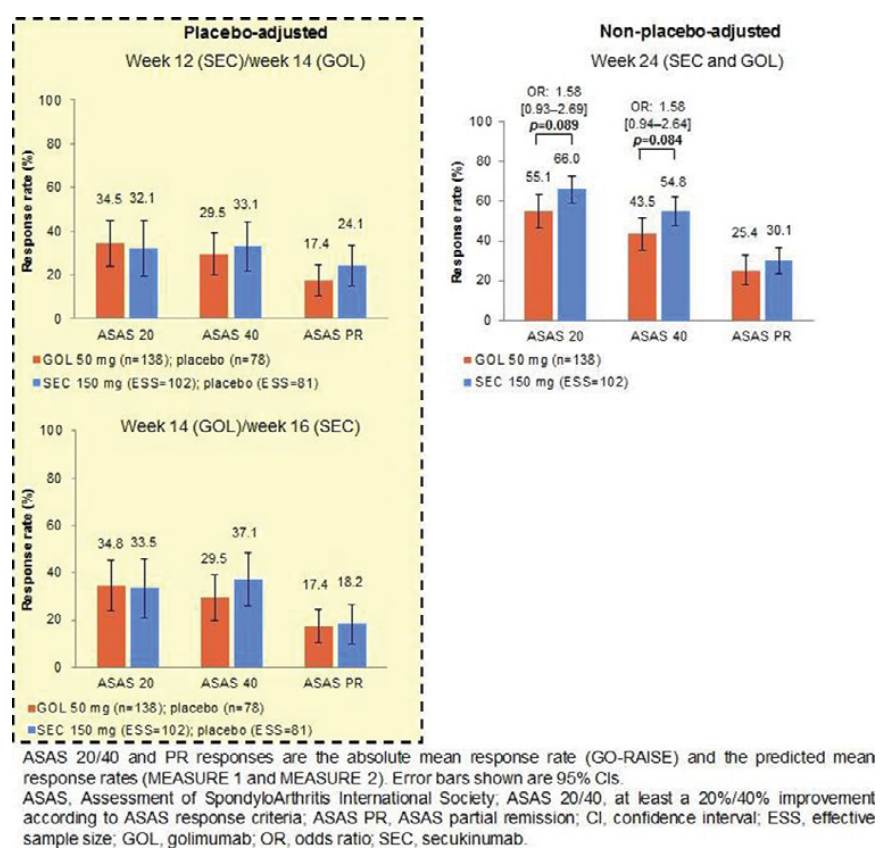
Aample size: ASLL, golimumab; OR, Odds ratio; SEC Secukinumab

Conclusions: There was no evidence of differences in ASAS responses between SEC and GOL in placebo-adjusted analyses. In non-placebo-adjusted analyses, SEC showed higher ASAS 20 and ASAS 40 responses than GOL at week 24.

References:

[1] Phillippo DM et al. (2016) NICE DSU Technical Support Document 18. Available from: http://www.nicedsu.org.uk.

Disclosure of Interest: W. Maksymowych Grant/research support from: AbbVie, Pfizer and Sanofi, Consultant for: AbbVie, Amgen, Eli Lilly, Janssen, Merck, Novartis, Pfizer, Sanofi and UCB, E. Choy Grant/research support from: Pfizer, Roche and UCB, Consultant for: Chugai Pharma, Eli Lilly, Janssen, Novartis, Pfizer, Regeneron, Roche, R-Pharm and Sanofi, Y. Yazici Grant/research support from: Celgene, BMS and Genentech, Consultant for: Celgene, BMS and Novartis, J. Walsh Consultant for: Novartis, H. Thom Consultant for: Eli Lilly, F Hoffman-La Roche, Novartis Pharma AG and Pfizer, C. Kalyvas Employee of: Paid employee of the Mapi Group. The Mapi Group received funding from Novartis Pharma AG for this study, T. Fox Employee of: Novartis employee with stock, K. Gandhi Employee of: Novartis employee with stock, S. Jugl Employee of: Novartis employee with stock

DOI: 10.1136/annrheumdis-2017-eular.5850

\section{AB0708 TRANSITION FROM ONGOING INFLIXIMAB REFERENCE PRODUCT TO ITS BIOSIMILAR: CAN WE TALK ABOUT A FAILURE?}

W. Akrout $^{1}{ }^{1}$, A. Bosycot ${ }^{1}$, R. Levet-Labry ${ }^{1}$, E. Gazaix-Fontaine ${ }^{2}$, M. Paul $^{1}$, P. Claudepierre ${ }^{2} .{ }^{1}$ Clinical Pharmacy Department; ${ }^{2}$ Rheumatology Department, Henri Mondor University Hospital AP-HP, Créteil, France

Background: Some recent publications support the fact that there is no clinically meaningful difference between infliximab biosimilar and the originator in naïve patients, but sole a few of them actually assessed the transition itself. Since May 2016, according to the European guidelines, the French Health Authorities have allowed interchangeability between a biotherapy reference product and its biosimilar. According to the last studies focusing on the safety and efficacy issues, avoiding the transition to biosimilar looks no longer justified.

Objectives: This work aimed to understand to which extent the use of infliximab biosimilar may result in the failure of the switch strategy in spondyloarthritis patients.

Methods: This is a retrospective study conducted from June to December 
2016. Only were included the adult patients with rheumatoid arthritis (RA), spondyloarthritis (SpA) or psoriatic arthritis (PsA) having accepted the transition from Infliximab reference product $\left(I F X^{R}\right)$ to its biosimilar $\left(I F X^{B}\right)$. For SpA patients, transition monitoring was based on the end-of-dose "wearing off" (WO) phenomenon assessment before and after the switch, disease activity score BASDAI and also the inflammatory marker CRP. Transition was considered as unsuccessful after $2 \mathrm{IFX}$ infusions with patient complain. In this case, the drug level was determined using ELISA before switching back to IFX ${ }^{R}$, and any spacing infusions because a concomitant health disorder was sought.

Results: Of the 99 patients treated with IFX $X^{R}$ for more than 2 years, $91 \%(90 / 99)$ accepted the transition: $53 \mathrm{SpA}, 23 \mathrm{RA}$ and 14 PsA. Fourteen patients (12 SpA, 2 RA) didn't reach the 3rd IFX ${ }^{B}$ administration. SpA patients reported the occurrence of arthralgia (12/12) and a partial (8/12) or total (4/12) efficacy loss. Only 5 patients reported a 2 point-increase or more regarding the BASDAI score, and only 3 patients had an increase in the CRP level (Cf.Table 1). Except for $\mathrm{P} 1$, the efficacy loss was associated in each case with WO phenomenon already reported before the transition (8/12) or undetectable IFX level (3/12). For these 3 last patients, the IFX $X^{B}$ drug administration rate was increased by at least 2 weeks than usual because of a concomitant infection.

Table 1: SpA patients discontinued IFXB outcomes

\begin{tabular}{|c|c|c|c|c|c|c|c|c|c|c|c|c|}
\hline SpA patients & P1 & P2 & P3 & P4 & P5 & P6 & P7 & P8 & P9 & P10 & P11 & P12 \\
\hline Arthralgia & + & + & + & + & + & + & + & + & + & + & + & + \\
\hline $\begin{array}{l}\text { Partial or total } \\
\text { efficacy loss }\end{array}$ & + & + & + & + & + & + & + & + & + & + & + & + \\
\hline TBASDAI & - & + & + & + & - & - & - & + & - & - & - & + \\
\hline T CRP & + & - & - & - & - & + & - & + & - & - & - & - \\
\hline Existing wo & - & + & + & - & + & + & + & - & + & + & - & + \\
\hline $\begin{array}{l}\text { Undetectable } \\
\text { IFX level }\end{array}$ & - & - & - & + & - & - & - & + & - & - & + & \\
\hline $\begin{array}{l}\text { Spacing } \\
\text { infusions }\end{array}$ & - & - & - & + & - & - & - & + & - & - & + & \\
\hline
\end{tabular}

Conclusions: This observational study showed a transition failure rate at $16 \%$ (76/90) in global, which reached $23 \%(12 / 53)$ if limited to SpA. All SpA patients with supposed transition failure reported either a disease escape beforehand or a concomitant infection requiring to spacing infusions. In order to complete these results, an anti-IFX antibodies monitoring is in progress so to highlight any IFX activity loss. Any failure observed with the transition would be actually more complex than the presumably inefficacy of the biosimilar IFX ${ }^{B}$ especially for $\mathrm{SpA}$ patients. So, it seems difficult to assess robustly the inefficacy of the transition to IFXB in SpA patients only according to patient complain.

\section{References:}

[1] Park W et al. Arthritis Res Ther. 2016 Jan 20;18:25.

[2] Fiorino G et al. Expert Rev Clin Immunol. 2016;12(4):361-3.

[3] Jacobs I et al. BioDrugs. 2016 Dec;30(6):525-570.

Disclosure of Interest: None declared

DOI: 10.1136/annrheumdis-2017-eular.4823

\section{Spondyloarthritis - clinical aspects (other than treatment)}

\section{AB0709 THE RELATIONSHIPS BETWEEN THORACIC REGION INVOLVEMENT AND FUNCTIONS OF UPPER EXTREMITY, SCAPULAR KINEMATICS IN PATIENTS WITH ANKYLOSING SPONDYLITIS: PILOT STUDY}

O. Mete ${ }^{1}$, D. Oskay ${ }^{1}$, A. Tufan ${ }^{2} .{ }^{1}$ Physiotherapy and Rehabilitation; ${ }^{2}$ Internal Medicine, Division of Rheumatology, Gazi University, Ankara, Turkey

Background: Ankylosing spondylitis (AS) is a chronic inflammatory rheumatic disease that mainly affects the axial skeleton. As the disease progresses, increased thoracic kyphosis can be seen in these patients. Because of increase in thoracic kyphosis, the orientation of the scapula on the thorax and thus the functions of upper extremity may change.

Objectives: The aim of the study is to investigate the relationships between thoracic region involvement and functions of upper extremity, scapular kinematics in patients with AS.

Methods: Fifteen (15) patients with AS and eleven (11) healthy control were participated in the study. Thoracic kyphosis angle and shoulder range of motions were assessed by using digital inclinometer, scapular and shoulder muscle strength were assessed by using digital dynamometer, three dimensional (3D) scapular kinematics were assessed by using electromagnetic tracking system, disability level of upper extremity were assessed by Turkish Version of Disability of Arm, Shoulder and Hand Questionnaire (DASH-T). Spearman correlation coefficient, Pearson correlation coefficient, Mann-Whitney U Test and Independent Sample T-test were used for statistical analysis.
Results: DASH-T, thoracic kyphosis angle, shoulder abduction, internal rotation, external rotation of dominant side, shoulder abduction, internal rotation of non-dominant side, anterior deltoid, middle deltoid, serratus anterior, downward trapezius muscle strengths of dominant and non-dominant side, upward rotation of scapula during $30,60,90$ degrees humerothoracic elevations at sagital plane of dominant and non-dominant side showed significantly differences between two groups. Thoracic kyphosis angle showed correlations with DASH-T $(p<0,05, r: 0,619)$, shoulder flexion, abduction, internal rotation, external rotation of dominant side $(p<0,05, r:-0,867),(p<0,05, r:-0,580),(p<0,05, r:-0,657),(p<0,05, r:-$ $0,599)$ and shoulder flexion, abduction, internal rotation of non-dominant side $(p<0,05, r:-0,813), \quad(p<0,05, r:-0,665), \quad(p<0,05, r:-0,741)$, respectively. Thoracic kyphosis angle showed correlations with anterior deltoid, middle deltoid, serratus anterior, middle trapezius, downward trapezius muscle strengths of dominant side and non-dominant side, respectively $(p<0,05, r:-0,899),(p<0,05, r$ :$0,854),(p<0,05, r:-0,805),(p<0,05, r:-0,791),(p<0,05, r:-0,633),(p<0,05, r:-0,877)$, $(p<0,05, r:-0,796),(p<0,05, r:-0,884),(p<0,05, r:-0,724),(p<0,05, r:-0,673)$. Correlations between thoracic kyphosis angle and anterior tilt of scapula during 90 degree humerothoracic elevations at sagital plane of dominant side were obtained $(p<0,05, r: 0,522)$.

Conclusions: Scapulothoracic joint biomechanics and functions of upper extremity were affected by kyphotic posture in patients with AS. One of the most important causes of biomechanical impairment in AS patients is the deterioration of scapular kinematics with kyphosis. For preventing functional impairment, treatment programs should be supplemented with scapular kinematic exercises. References:

[1] Braun (2007). Ankylosing spondylitis. The Lancet.

[2] Kebaetse (1999). Thoracic position effect on shoulder range of motion, strength, and three-dimensional scapular kinematics. Archives of physical medicine and rehabilitation.

Disclosure of Interest: None declared

DOI: 10.1136/annrheumdis-2017-eular.5869

\section{AB0710 REVIEW OF PREGNANCY OUTCOMES IN SPONDYLOARTHROPATHY IN A UNIVERSITY TEACHING HOSPITAL}

A. Shajpal ${ }^{1}$, M. Khare ${ }^{2}$, A. Moorthy ${ }^{3} \cdot{ }^{1}$ Obstetrics; ${ }^{2}$ Maternal Fetal Medicine; ${ }^{3}$ Rheumatology, University Hospitals of Leicester, Leicester, United Kingdom

Background: Spondyloarthropathy $(\mathrm{SpA})$ is a chronic inflammatory condition of the spine affecting mainly the male population however the incidence amongst the female population is increasing. The peak incidence of $\mathrm{SpA}$ is in the reproductive age group. There has been a lack of focus on pregnancy in SpA as compared to other autoimmune condition such as Lupus and Rheumatoid arthritis, but this is changing. There is a paucity of information (1) on fertility and pregnancy outcomes in this condition compared to other diseases and this may lead to inequality in healthcare delivery.

Objectives:

- To review the pregnancy outcomes in women with SpA in our unit

- To review ankylosing spondylitis activity during pregnancy

- To improve the quality of care in this group of patients by developing local pathways and appropriate MDT involvement

Methods: This is a retrospective case review of pregnancies in women with $\mathrm{SpA}$ booked at a large tertiary teaching hospital over three years between January 2014 and December 2016. We have an annual delivery rate of 11,000 maternities. The maternity electronic database and clinic diaries were used to identify the cases. A standardised proforma was used to collect and collate the data for demographics, pre pregnancy counselling, disease activity and pregnancy outcome.

Results: Six pregnancies were identified in the study period. All patients were under the care of a Rheumatologist. The maternal age range was between 28 and 35 years. The BMI ranged between 18 and 37 . Ethnicity included 5 caucasian and one Asian woman. Five women had previous pregnancies and one was in her first pregnancy. Two of the multiparous women had previously delivered by caesarean section. Three of the six women suffered from anxiety and/or depression and one had fibromyalgia. Two of the six patients were not on any medication at the start of pregnancy and didn't require any during pregnancy. Four women needed various analgesics and one patient was on sulfasalazine but stopped this at 5 weeks' gestation. NSAIDs was stopped in 3 women after confirmation of pregnancy. One patient who was on Anti TNF therapy discontinued the drug preconception. We observed $50 \%$ attended specialist maternal-fetal medicine and anaesthetic services. One patient saw a physiotherapist and accessed hydrotherapy during pregnancy.

Two of six patients delivered preterm ( $<37$ weeks) and 4 delivered at term ( $>37$ weeks). Of the preterm deliveries, 1 went into spontaneous labour not related to disease flare and the other was delivered electively for fetal concerns. All the women delivered by caesarean section. One was planned as an elective caesarean for maternal request due to difficulty abducting legs. All the remaining caesarean deliveries were for obstetric indications not related to SpA.

Conclusions: This small observational case series did not highlight any worsening SpA disease activity or poor pregnancy outcome. Further larger studies are required. However a care pathway for managing this group of patients would help to standardise the care during pregnancy. A multidisciplinary approach is essential to optimise the quality of care for these patients. 